\title{
Positron annihilation study in polyaniline conducting polymer
}

\author{
Z.L. PENG, S.Q. LI, Y.Q. DAI, B. WANG, S.J. WANG, H. LIU* and H.Q. XIE*
}

Department of Physics, Wuhan University, Wuhan 430072, P.R. of China

* Dept. of Chem., Huazhong Univ. of Sci. \& Tech., Wuhan 430072, P.R. of China

\begin{abstract}
The positron lifetime spectra in polyaniline have been measured as a function of protonation level ( $\mathrm{pH}$ from 7 to -0.8 ). We observed that (1) the short lifetime $\tau_{1}$ decreased with the protonation level; (2) the intermediate lifetime was constant ( $\left.\tau_{2} * 360 \mathrm{ps}\right)$, whereas its intensity $I_{2}$ increased with increasing protonation level which was related to the conductivity of material. These results are discussed in terms of conducting island model.
\end{abstract}

\section{INTRODUCTION}

The polyaniline family of polymers have been the focus of recent investigation due to the special characteristics and application $[1,2]$. Series corresponding variation in physical properties with the protonation level has been reported [3]. In order to understand the nature of physical process which occur in polyaniline due to doping, we performed the positron lifetine measurement as a function of protonation level and discussed its sensitivity to the microstructural changes in the polymer matrix.

\section{BXPERTMENT}

Polyaniline powder was prepared by chemical synthesis as described by Macdiamid et al [1]. The powder obtained was extracted with $\mathrm{CH}_{3} \mathrm{CN}$, dried and then equilibrated with constant stirring in $0.1 \mathrm{M} \mathrm{NH}_{4} \mathrm{OH}$ for $24 \mathrm{~h}$ giving deprotonated. The polyaniline powder with different protonation states were obtained by treatment of its base form of powder with different acidity aqueous $\mathrm{HCl}$ solution and compressed into $1 \mathrm{~mm}$ thickness pellets under $150 \mathrm{~kg} / \mathrm{cm}^{2}$ and its conductivity was measured by four-probe method.Positron lifetime spectra were measured using a fast-fast coincidence system with time resolution of $245 \mathrm{ps}$ and each spectrum contained one million counts. 
The variation of the conductivity of polyaniline powder at room temperature with the protonation state is shown in fig.1.The emeraldine base form of polyaniline has a conductivity of $10^{-10}$ ${ }^{10} \mathrm{~s} / \mathrm{cm}$. Through treatment with aqueous $H C 1$ of $\mathrm{pH}$ varying from 7 to -0.8 , protons were added to a fraction of formerly unprotonated nitrogen sites, while $\mathrm{Cl}^{-}$remain interstitially for charge neutrality. Though the number of electrons on the polymer chain remains constant, the conductivity increased to $3 \mathrm{~s} / \mathrm{cm}$. At higher degree of the protonation states( $\mathrm{pH} \leq 1)$, the conductivity reaches a maximum value and does not change with the varying $\mathrm{pH}$. Therefore, an insulator to metal transition for samples was observed.

\section{(2). Positron annihilation parameter}

Positron spectrum of polyaniline has been best resolved into three component by using PATFIT program [4]. Since the intermediate lifetimes almost remain $360 \mathrm{ps}$, so we fixed t? at $360 \mathrm{ps}$ to reduce the statistical error.

As we know, the short lifetime $\tau_{1}$ and the intermediate lifetime $\tau_{2}$ are attributed to positron annihilation mainly from the bulk and trapped state, respectively , and the long-lived component $\left(\tau_{3}=1.93_{ \pm} 0.12 \mathrm{~ns}, I_{3}=2.2_{ \pm} 0.3 \%\right)$ is due to o-Ps annihilation on the surface of the material which is nearly independent to protonation level. The $\tau_{1}$ decreases with the protonation level, whereas $I_{2}$ first increases with the protonation level and then remains constant for higher doping level(pHsl), as shown in fig.2 and 3 . The decrease of the $\tau_{1}$ with increase of protonation level indicated the increase of the electron density in bulk of material.Comparing fig. 1 and fig.3, intermediate intensity $I_{q}$ is obviously related to the conductivity of our sample,so we pay the special attention to the variation of the intermediate component with respect to protonation level.

In the base form of polyaniline, the trapping sites might be voids in the amorphous regions and not-totally collapsed chlorine anion vacancies left by deprotonating. In protonated emeraldine, the localized chlorine dopant anions should perturb the intermolecular force in its vicinity and create local lattice distortions in a manner so as to create local negatively charged regions which will trap the positrons[5]. The increase amount of the trapping sites in sample is essentially determined by the concentration of the chlorine dopant anion.According to the model of conducting island [2], in polyaniline,partial protonation leads to phase segregation into metallic (fully protonated) regions and insulating (unprotonated) regions. The anount of metallic regions increases with the protonation level, and the formation of large amount of metallic regions will reduce potential barrier and leads to the increase in conductivity. The increase of $I_{2}$ is just an indicator of the metallic regions, so as the equilibriun $\mathrm{pH}$ of aqueous $\mathrm{HCl}$ solution decreases, the increase of $\mathrm{I}_{q}$ has the same tendency as the conductivity. The larger the $I_{2}$ value, the greater is the conductivity. 


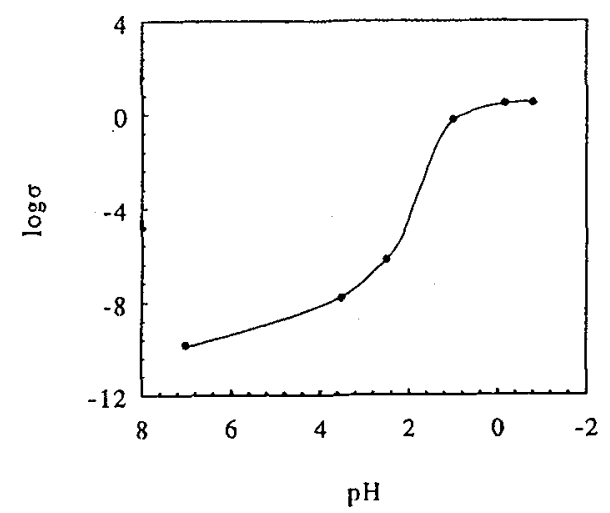

Fig. 1 The variation of conductivity with protonation level in polyaniline

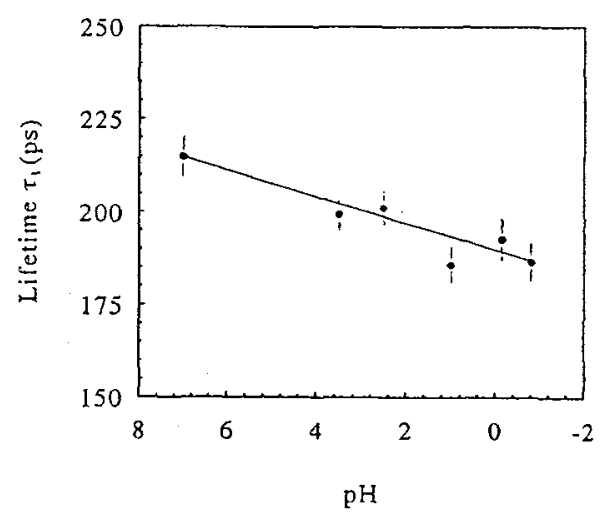

Fig.2 The positron lifetime $\tau_{1}$ against protonation level in polyaniline

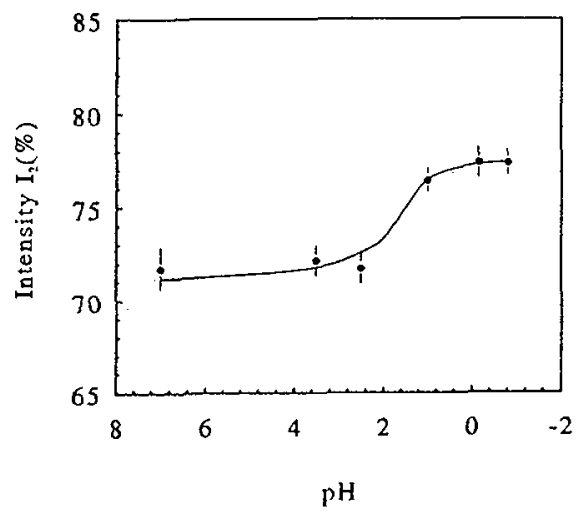

Fig. 3 The positron intensity $I_{2}$ against protonation level in polyaniline

\section{RBEFERENCES}

11/ J.C.Chiang, A.G.MacDiarmid, Synth.Met.13(1986) 193

/2/ A.J.Epstin,A.G.MacDiarnid, Mol.Cryst.Liq.Cryst 160(1988)165

/3/ F.Zuo, M. Angelopoulos et al, Phys.Rev.B 36(1987) 3475

/4/ K.Kirkegaad and M.Eldrup, Computer Phys.Commun 1(1974)401

15/ S.C.Sharma, S.Krishnamoorthy et al, Fhys.Rev.B 41(1990)5258 